\title{
HL2, an Inconsistency-adaptive and Inconsistency-resolving Logic for General Statements that might have Exceptions.
}

\author{
Guido Vanackere* \\ Center for Logic and Philosophy of Science \\ University of Ghent \\ Guido.Vanackere@rug.ac.be
}

\begin{abstract}
The present paper offers a new approach to non-monotonic logics and their reconstruction in terms of inconsistency-adaptive logics. By applying a special technique, universally quantified formulas are assigned instances that, given the paraconsistent framework, do not cause triviality even if they conflict with knowledge deriving from other sources. From the special instances, the usual instances may be derived conditionally, viz. provided they are not contradicted by statements derived with a higher preference ranking.
\end{abstract}

\section{Introduction}

In this paper I present a new approach to non-monotonic reasoning. The underlying idea is that universally quantified formulas (henceforth UQF) are formulated unrestrictedly, even if they have exceptions, but the derivation of instances is restricted, and derived instances are not finally derivable (i.e. do not belong to the consequence set), when the instance is known to concern an exception to the UQF.

The effect is realized by recurring to an inconsistency-adaptive logic HL2 that is based on a specific paraconsistent logic pHL2. According to pHL2,

${ }^{*}$ I am indebted to the two referees; their remarks enabled me to improve the exposition at several points. I thank Kristof De Clercq; his experience with problems concerning default-reasoning and circumscription, has been very useful for me (see his [6]). I am especially obliged to Diderik Batens; his work on adaptive logics and his assistance in the writing of this paper were indispensable. 
a UQF $(\forall \alpha) A(\alpha)$ unconditionally entails $\sim \neg A(\beta)$ in which " $\sim$ " is a paraconsistent negation and " $\neg$ " classical negation. According to HL2, $\sim A(\beta)$ entails $A(\beta)$, provided $\neg A(\beta)$ 'behaves consistently' (in a sense specified later) on the premises. I shall first clarify the role and the effects of paraconsistent and inconsistency-adaptive logics, and then motivate the transition to the logics presented below.

Paraconsistent logics avoid that inconsistent theories are turned into trivial ones. A straightforward strategy to obtain a paraconsistent logic consists in weakening classical logic $(\mathbf{C L})$ by dropping one half of the meaning of negation, reducing it to "If $A$ is false, then $\sim A$ is true". In [4] this paraconsistent logic is called $\mathbf{C L u N}{ }^{1} \mathbf{C L u N}$ indeed allows for non-trivial inconsistent theories. It does so however by eliminating too many inferences: we want the intuitivily correct consequences to be derivable when no inconsistency is involved; e.g. we want to apply Disjunctive Syllogism $(A \vee B, \sim A$ ( $B$ ) whenever $A$ does not behave inconsistently. The inconsistency-adaptive logics ACLuN1 and ACLuN2, based on CLuN, enable us to do so - see [2], [3] and [4]. ${ }^{2}$ In these logics some consequences which are CL-derivable but not CLuN-derivable, are ACLuN-derivable, provided certain formulas are reliable - that is: do not behave inconsistently in view of the premises.

Although inconsistency-adaptive logics isolate inconsistencies and deliver all classical consequences whenever the specific inconsistencies do not prevent this, they are not fully adequate with respect to everyday reasoning. Indeed, in many situations we want to eliminate the inconsistencies as they occur. If an inconsistency turns up, for instance within our most reliable knowledge, we often wish to reject one half of the inconsistency and to retain the other. The two kinds of inconsistencies considered in the present paper are caused by the fact that we have different ways to obtain information about the same statement. The information may stem from observation, possibly combined with logical deduction. It may also be derived from general knowledge, for example from UQFs - and remark that a combination

\footnotetext{
${ }^{1}$ In [2] and [3] this paraconsistent logic was called PIL. It was renamed in view of the creation of analogous logics dealing with other abnormalities. The $\mathbf{N}$ stands for Negation, the $\mathbf{u}$ stands for gluts. CLaN, for instance, is a paracomplete logic; here the a stands for gaps.

CLuN is obtained from CL by dropping the axiom $(A \supset B) \supset((A \supset \sim B) \supset \sim A)$. Classical negation, $\neg$, is introduced by $\neg A={ }_{\mathrm{df}} A \supset \perp$. Remark that neither Replacement of Identials nor Replacement of Equivalents hold in the scope of a " ". Also, CLuN maximally isolates inconsistencies; from $A \& \sim A$, no other inconsistency is derivable, not even about subwffs or superwffs of $A$.

${ }^{2}$ In [2] and [3], these logics were called APIL1 and APIL2.
} 
of UQFs may lead to conflicting information. In view of the elegance and power of our most reliable knowledge, we are interested in general statements without exceptions. If conflicts arise, we want to reject the instance of the general statement and to retain the more specific information (i.e. the observational information or the information derived from more specific UQFs).

In the second part of this paper I introduce Hypothesis Logic HL. This adaptive logic is based on $\mathbf{p H L}$, which is obtained from $\mathbf{C L}$ by modifying its instantiation rule: from $(\forall \alpha) A(\alpha), \sim \neg A(\beta)$ is derived instead of $A(\beta)$. The curl is the paraconsistent negation as defined in CLuN. As an immediate result, the inconsistency arising if $\neg A(\beta)$ is provided by information deriving from a different source, does not cause explosion. According to $\mathbf{H L}, A(\beta)$ is only derivable from $\sim \neg A(\beta)$ provided $\neg A(\beta)$ is not derivable from the available premises. The modification has a further and most interesting effect: if an exception turns out to be derivable after the instance $A(\beta)$ was conditionally derived, the conditionally derived instance will not be considered as finally derivable because the line in which it occurs is marked in view of a strictly logical marking rule. The advantage of such a marking rule is clear: when inconsistencies are weeded out within a proof, we obtain a richer (but not trivial) and (in as far as we eschew inconsistencies) more interesting consequence set, and we do so by strictly formal means.

In the third part of the paper I introduce HL1, a logic pertaining to languages that combine the universal quantifier of $\mathbf{H L}$ with the one of CL. Unlike HL, HL1 enables us also to resolve inconsistencies deriving from UQFs. The idea is that 'classical' UQFs can be instantiated unconditionally, while the others can only be conditionally instantiated. In order to apply HL1, we need to classify (from the very outset) UQFs in classical and 'exceptional' ones - this elimination of inconsistencies is not strictly logical but relies on non-logical considerations.

In the final part I introduce HL2, which generalizes the idea of sorted quantifiers. Where HL1 contains one kind of exceptional universal quantifier, HL2 contains a preferentially ordered (indefinitely large) amount of them. For simplicity's sake, I shall only consider linear orderings. The preference of a UQF will be indicated by a number superscripted to the universal quantifier, lower numbers indicating the higher preferences (for the sake of generality, classical UQFs will receive the superscript 0 ). The relation between a HL2-UQF of preference $i$ and a HL2-UQF of preference $i+j(j \geq 1)$, is the same as the relation between a classical HL1-UQF and an exceptional HL1-UQF. Where a contradiction arises between (a formula 
derived from) an instance of a UQF of preference $i$ and (a formula derived from) an instance of a UQF of preference $i+j$, it will follow from the occurrence of the former that the line in which the latter occurs is marked, and hence the latter will not be finally derivable.

\section{HL.}

In HL all UQFs may have exceptions. This implies that the CL-instances are derivable conditionally. Where no exception occurs, the conditions are not overruled and the $\mathbf{H L}$-instances are the $\mathbf{C L}$-instances.

All negations occurring in natural languages are formalized by means of " $\neg$ ", the classical negation, but the language scheme of HL contains a second, paraconsistent negation " $\sim$ ". The universal instantiation rule of $\mathbf{H L}$ is: ${ }^{3}$

UI: $(\forall \alpha) A(\alpha) / \sim \neg A(\beta)$

The instance can be read as: there is no reason to accept (= " $\sim$ ") that $A(\beta)$ is not the case (= " $\neg ")$. An immediate advantage of this instantiantion rule is that no (classical) inconsistency occurs when $\neg A(\beta)$ is also derivable from the premises. In the underlying paraconsistent logic $\mathbf{p H L}$ it is impossible to derive $A$ from $\sim \neg A$; in $\mathbf{H L}$, the adaptive logic based on it, $A$ can be derived from $\sim \neg A$ conditionally. All pHL-consequences are HL-consequences. Some CL-consequences which are not pHL-derivable, are $\mathbf{H L}$-derivable. An example of a $\mathbf{H L}$-proof:

$$
\begin{array}{llll}
(\forall x)(A x \supset(P x \& \neg Q x)) & - & \text { PREM } & - \\
B a \supset Q a & - & \text { PREM } & - \\
A a & - & \text { PREM } & - \\
B a & - & \text { PREM } & - \\
\sim \neg(A a \supset(P a \& \neg Q a)) & 1 & \mathrm{UI} & - \\
A a \supset(P a \& \neg Q a) & 5 & \mathrm{CDN}^{4} & \neg(A a \supset(P a \& \neg Q a)) \\
P a \& \neg Q a & 3,6 & \mathrm{MP} & \neg(A a \supset(P a \& \neg Q a)) \\
\neg Q a & 7 & \mathrm{SIM} & \neg(A a \supset(P a \& \neg Q a)) \\
Q a & 2,4 & \mathrm{MP} & -
\end{array}
$$

\footnotetext{
${ }^{3}$ In this paper the proof theory is formulated axiomatically. In actual proofs however it is handier to make use of Fitch-style rules. I skip the proofs of the derivation of the Fitch-style rules from the axioms. (These proofs are standard.) Where necessary the applied Fitch-style rules are explained in a footnote.

${ }^{4} \mathrm{CDN}$ stands for conditional double negation. This rule is explained in Section 2.2.1.
} 
The formula in line (6) is derived on condition of the $\sim$-consistent behaviour of the formula in the fifth element of line (6). Every formula derived from a conditionally derived formula takes over the condition(s). If we stop the proof at line (9), we have derived both $Q a$ and $\neg Q a$, which is not exactly what we want; as $Q a$ is derived from more specific information than $\neg Q a$, we want only $Q a$ to be finally derivable. If we continue the proof, we find out that the condition mentioned in lines (6)-(8) is overruled.

$\begin{array}{llll}\neg \neg Q a & 9 & \mathrm{DN} & - \\ \neg P a \vee \neg \neg Q a & 10 & \mathrm{ADD} & - \\ \neg(P a \& \neg Q a) & 11 & \mathrm{ND} & - \\ \neg(A a \supset(P a \& \neg Q a)) & 3,12 & \mathrm{NI}^{5} & -\end{array}$

As both $\neg(A a \supset(P a \& \neg Q a))$ (line (13)) and its negation (line (5)) are derived unconditionally, the condition in lines (6)-(8) is overruled, and therefore the formulas in these lines will not be considered as finally derivable, i.e. $Q a$ belongs to the consequence set of the premises $\left(C n_{\mathbf{H L}}(\{\text { premises }\})\right)^{6}$, while $\neg Q a$ does not. The inconsistency is resolved. I will introduce a derived marking rule by which line (8) can be marked without extending the proof with lines (9)-(13). In what follows lines of a proof that contain an overruled condition in their fifth element, will be marked with $\dagger$, e.g. lines (6)-(8) in the proof above. Marked lines do not belong to the proof anymore. Formulas in the second element of marked lines are not HL-consequences.

The notation " $\sharp A$ " will be the short for " $A \& \sim A$ ".

\subsection{The underlying paraconsistent logic pHL.}

\subsubsection{Proof Theory of pHL.}

The axiom scheme of pHL is composed of a suitable set of axioms for $\supset$, $\&, \vee, \equiv, \perp, \neg, \exists$ and $=^{7}$ (as for $\mathbf{C L}$ ), together with:

\footnotetext{
${ }^{5}$ NI stands for negation of the implication. In view of the fact that $A \supset B$ is true iff $A$ is not true (or $\neg A$ is true) or $B$ is true, $\neg(A \supset B)$ is true iff $A$ is true and $B$ is not true (or $\neg B$ is true).

${ }^{6}$ Where $\mathbf{X L}$ is a logic, and $\Gamma$ is a set of well formed formulas, $C n_{\mathbf{X L}}(\Gamma)=\left\{A \mid \Gamma \vdash_{\mathbf{X L}}\right.$ $A\}$.

${ }^{7}$ Replacement of identials is restricted as follows:

$(\mathbf{A = 2}) \alpha=\beta \supset(A \supset B)$ where $B$ is obtained by replacing in $A$ an occurrence of $\alpha$ that occurs outside the scope of $a \sim b y \beta$.
} 
$\mathbf{A} \forall: \quad(\forall \alpha) A(\alpha) \supset \sim \neg A(\beta)$

$\mathbf{R} \forall: \quad$ To derive $\vdash A \supset(\forall \alpha) B(\alpha)$ from $\vdash A \supset \sim \neg B(\beta)$, provided $\beta$ does not occur in either $A$ or $B(\alpha)$

$\mathbf{A} \sim: \quad A \vee \sim A$

Replacement of equivalents and replacement of identials is restricted to subformulas outside the scope of $\sim$. As $A \vdash_{\mathrm{pHL}} \sim \neg A$, the usual $\mathbf{R} \forall$ rule of CL is derivable. Remark that $(A \& \sim A) \supset B$ is not a pHL-theorem. In view of the axiom scheme, $A$ is not pHL-derivable from $\sim \neg A$, and $A(\beta)$ is not derivable from $(\forall \alpha) A(\alpha)$. All CL-theorems concerning $\supset, \&, \vee, \equiv, \perp$, and $\neg$ are valid in $\mathbf{p H L}$.

The $\sim$-paraconsistent logic $\mathbf{p H L}$ provides us with the first property every logic for general statements that might have exceptions should have: the occurrence of an exception does not cause triviality. In $\mathbf{H L}$, the adaptive logic based on pHL, $A(\beta)$ will be derivable from $(\forall \alpha) A(\alpha)$ whenever $\neg A(\beta)$ is not derivable. The following theorems show that usual CL-consequences are derivable in pHL in disjunction with a $\sim$-inconsistency.

Theorem 1 If $\Gamma \vdash_{\mathrm{pHL}} \sim \neg A$, then $\Gamma \vdash_{\mathrm{pHL}} \sharp \neg A \vee A$.

Proof. As $\neg A \vee A$ is a pHL-theorem, $\sim \neg A \vdash_{\mathrm{pHL}} \sharp \neg A \vee A$. $\square$.

Theorem 2 If $\Gamma \vdash_{\mathbf{p H L}} A \supset B$ and $\Gamma \vdash_{\mathbf{p H L}} \sim \neg A$, then $\Gamma \vdash_{\mathbf{p H L}} \sharp \neg A \vee B$.

Proof. The proof follows immediately from Theorem 1 and $A \supset B, C \vee A$ $\vdash_{\mathrm{pHL}} C \vee B . \square$.

\subsubsection{Semantics of pHL.}

Let $\mathcal{S}$ be the set of sentential letters, $\mathcal{P}^{n}$ the set of predicative letters of rank $n, \mathcal{C}$ and $\mathcal{V}$ the set of letters for individual constants and variables respectively, $\mathcal{F}$ the set of (open and closed) formulas, $\mathcal{W}$ the set of well formed formulas (wffs), and $\mathcal{N}=\{\sim A \mid A \in \mathcal{F}\}$. Let the members of $\mathcal{C}$ as well as the members of $\mathcal{V}$ be given in a certain order denoted by " $<$ ". The following wffs will be called primitive: members of $\mathcal{S}$, formulas of the form $\alpha=\beta$, and primitive predicative formulas.

A model is a couple $M=\langle D, v\rangle$ in which $D$ is a set and $v$ is an assignmentfunction defined by:

$\mathrm{S} 1.1 \mathrm{v}: \mathcal{S} \longrightarrow\{0,1\}$

$\mathrm{S} 1.2 \mathrm{v}: \mathcal{C} \cup \mathcal{V} \longrightarrow \mathrm{D}$ such that $\mathrm{D}=\{\mathrm{v}(\alpha) \mid \alpha \in \mathcal{C} \cup \mathcal{V}\}$

$\mathrm{S} 1.3 \mathrm{v}: \mathcal{P}^{n} \longrightarrow P\left(\mathrm{D}^{n}\right)$ (the power set of the $n$-th Cartesian product of $\mathrm{D}$ )

$\mathrm{S} 1.4 \mathrm{v}: \mathcal{N} \longrightarrow\{0,1\}$ 
The valuation-function $v_{M}$ determined by the model $M$ is defined as follows:

$\mathrm{S} 2.1 \mathrm{v}_{\mathrm{M}}: \mathcal{F} \longrightarrow\{0,1\}$

S2.2 where $A \in \mathcal{S}, \mathrm{v}_{\mathrm{M}}(A)=\mathrm{v}(A)$

$\mathrm{S} 2.3 \quad \mathrm{v}_{\mathrm{M}}\left(\pi^{n} \alpha_{1} \ldots \alpha_{n}\right)=1$ iff $\left\langle\mathrm{v}\left(\alpha_{1}\right), \ldots \mathrm{v}\left(\alpha_{n}\right)\right\rangle \in \mathrm{v}\left(\pi^{n}\right)$

$\mathrm{S} 2.4 \mathrm{v}_{\mathrm{M}}(\alpha=\beta)=1$ iff $\mathrm{v}(\alpha)=\mathrm{v}(\beta)$

$\mathrm{S} 2.5 \mathrm{v}_{\mathrm{M}}(\neg A)=1$ iff $\mathrm{v}_{\mathrm{M}}(A)=0$

$\mathrm{S} 2.6 \quad \mathrm{v}_{\mathrm{M}}(\sim A)=1$ iff $\mathrm{v}_{\mathrm{M}}(A)=0$ or $\mathrm{v}(\sim A)=1$

$\mathrm{S} 2.7 \quad \mathrm{v}_{\mathrm{M}}(A \supset B)=1$ iff $\mathrm{v}_{\mathrm{M}}(A)=0$ or $\mathrm{v}_{\mathrm{M}}(B)=1$

$\mathrm{S} 2.8 \mathrm{v}_{\mathrm{M}}(A \& B)=1$ iff $\mathrm{v}_{\mathrm{M}}(A)=1$ and $\mathrm{v}_{\mathrm{M}}(B)=1$

$\mathrm{S} 2.9 \mathrm{\vee}_{\mathrm{M}}(A \vee B)=1$ iff $\mathrm{v}_{\mathrm{M}}(A)=1$ or $\mathrm{\vee}_{\mathrm{M}}(B)=1$

$\mathrm{S} 2.10 \mathrm{v}_{\mathrm{M}}(A \equiv B)=1$ iff $\mathrm{v}_{\mathrm{M}}(A)=\mathrm{v}_{\mathrm{M}}(B)$

$\mathrm{S} 2.11 \mathrm{v}_{\mathrm{M}}((\forall \alpha) A(\alpha))=1$ iff $\mathrm{v}_{\mathrm{M}}(\sim \neg A(\beta))=1$ for all $\beta \in \mathcal{C} \cup \mathcal{V}$

$\mathrm{S} 2.12 \mathrm{v}_{\mathrm{M}}((\exists \alpha) A(\alpha))=1$ iff $\mathrm{v}_{\mathrm{M}}(A(\beta))=1$ for at least one $\beta \in \mathcal{C} \cup \mathcal{V}$

Definition: $\Gamma /{ }_{\mathrm{pHL}} A$ iff, for any $\mathbf{p H L}$-model $\mathrm{M}, \mathrm{v}_{\mathrm{M}}(B)=0$, for some $B \in \Gamma$ or $\mathrm{v}_{\mathrm{M}}(A)=1$.

Definition: A pHL-model is $\mathcal{N}$-normal iff $\mathrm{v}(\sim A)=0$ for all $A \in \mathcal{F}$.

Definition: Two models (even from different types of semantics) are equivalent iff they verify and falsify the same formulas.

The CL-models are these pHL-models that fulfil:

(1) $\mathrm{v}_{\mathrm{M}}(\sim A)=1$ iff $\mathrm{v}_{\mathrm{M}}(A)=0$.

(2) $\mathrm{v}_{\mathrm{M}}((\forall \alpha) A(\alpha))=1$ iff $\mathrm{v}_{\mathrm{M}}(A(\beta))=1$ for all $\beta \in \mathcal{C} \cup \mathcal{V}$.

In view of $(1), \mathrm{v}_{\mathrm{M}}(\sim A)=\mathrm{v}_{\mathrm{M}}(\neg A)$ in all CL-models $\mathrm{M}$.

Theorem 3 Any pHL-model that is equivalent to a $\mathcal{N}$-normal model, is equivalent to a $\mathbf{C L}$-model, and any $\mathbf{C L}$-model is equivalent to a $\mathcal{N}$-normal model.

Proof. If $\mathrm{M}$ is $\mathcal{N}$-normal, then $\mathrm{v}(\sim A)=0$, and hence, in view of $\mathrm{S} 2.6$, (1) is fulfilled. Hence $\sim$ is exactly the same as $\neg$, and can be replaced by it. Therefore, in view of $\mathrm{S} 2.11$ and $\mathrm{v}_{\mathrm{M}}(\neg \neg A)=\mathrm{v}_{\mathrm{M}}(A),(2)$ is fulfilled too. All other CL-clauses are the same as the corresponding $\mathbf{p H L}$-clauses. This shows that any $\mathcal{N}$-normal model is equivalent to a CL-model. Conversely, any CL-model is transformed into an equivalent $\mathcal{N}$-normal model by adding clause S1.4 and stipulating that $\vee(\sim A)=0$ for all $A \in \mathcal{F}$, and by adding clause S2.6. 


\subsubsection{Metatheory of pHL.}

In view of Theorem $3, \mathbf{C L}$ is modelled by the set of pHL-models that are equivalent to $\mathcal{N}$-normal -models, in other words, by $\sim$-consistent pHLmodels (those in which $\mathrm{v}_{\mathrm{M}}(\sim A)=\mathrm{v}_{\mathrm{M}}(\neg A)$ for any $\left.A \in \mathcal{F}\right)$.

Theorem 4 If $\Gamma \vdash_{\mathrm{pHL}} A$, then $\Gamma \models_{\mathrm{pHL}} A$.

(Proof left to reader.)

Theorem 5 If $\Gamma \models_{\mathrm{pHL}} A$, then $\Gamma \vdash_{\mathrm{pHL}} A$.

Proof. Let $\Gamma \models_{\mathrm{pHL}} A$ and suppose $\Gamma \nvdash_{\mathrm{pHL}} A$. We define a maximally non-trivial, deductively closed superset $\Delta(\subset \mathcal{W})$ of $\Gamma$ such that $A \notin \Delta$, and show that $\Delta$ defines a pHL-model of $\Gamma$. Let $B_{1}, B_{2}, \ldots$ be an enumeration of the members of $\mathcal{W}$ in which every wff of the form $(\exists \alpha) C(\alpha)$ is followed immediately by $C\left(\beta_{i}\right)$, where $\beta_{i}$ does not occur in $\Gamma, A$, or in any previous wff of the enumeration. The way to obtain such enumeration is standard. We define

$$
\begin{aligned}
& \Delta_{0}=C n_{\mathbf{p H L}}(\Gamma) \\
& \Delta_{i+1}=C n_{\mathbf{p H L}}\left(\Delta_{1} \cup\left\{B_{i+1}\right\}\right) \text { if } A \notin C n_{\mathbf{p H L}}\left(\Delta_{1} \cup\left\{B_{i+1}\right\}\right) \\
& \Delta_{i+1}=\Delta_{i} \text { otherwise } \\
& \begin{array}{c}
\Delta=\Delta_{0} \cup \Delta_{1} \cup \ldots
\end{array}
\end{aligned}
$$

It is easily seen that $\Gamma \subseteq \Delta$, that $A \notin \Delta$, and that $\Delta$ is deductively closed. $\Delta$ is maximally non-trivial. To see this, remark first that $A \supset C \in \Delta$ for all $C$. Indeed, if $A \supset C \notin \Delta$, there is a $\Delta_{i}$ such that $\Delta \cup\{A \supset C\} \vdash_{\mathrm{pHL}} A$; hence $\Delta_{i} \vdash_{\mathrm{pHL}}(A \supset C) \supset A$ by the deduction theorem; hence in view of the axiom $((A \supset B) \supset A) \supset A, \Delta_{i} \vdash_{\mathrm{pHL}} A$, which is impossible. If $E \notin \Delta$, then there is a $\Delta_{i}$ such that $\Delta_{i} \cup\{E\} \vdash_{\text {pHL }} A$; as $A \supset C \in \Delta$ for all $C$, $\Delta \cup\{E\}$ is trivial.

We define a $\mathbf{p H L}$-model $\mathrm{M}=\langle\mathrm{D}, \mathrm{v}\rangle$ as follows:

(1) $\mathrm{D}=\{\alpha \mid \alpha \in \mathcal{C}$ (and there is no $\beta \in \mathcal{C}$ such that $\beta<\alpha$ and $\alpha=\beta \in \Delta\}$,

(2) for all $C \in \mathcal{S}, \mathrm{v}(C)=1$ iff $C \in \Delta$,

(3) for all $\alpha \in \mathcal{C}$, if $\alpha \in \mathrm{D}, \mathrm{v}(\alpha)=\alpha$, if $\alpha \in \mathcal{C}-\mathrm{D}, \mathrm{v}(\alpha)$ is the $\beta \in \mathrm{D}$ such that $\alpha=\beta \in \Delta$ (there is a unique such $\beta$ by the definition of $\mathrm{D}$ ),

(4) for all $\pi \in \mathcal{P}^{n}, \mathrm{v}(\pi)=\left\{\left\langle\alpha_{1}, \ldots, \alpha_{n}\right\rangle \mid \pi \alpha_{1} \ldots \alpha_{n} \in \Delta\right\}$,

(5) for all $\sim C \in \mathcal{N}, \vee(\sim C)=1$ iff $C, \sim C \in \Delta$,

(6) each $\alpha \in \mathcal{V}$ is arbitrarily associated with a constant $\beta$ to the effect that $\mathrm{v}(\alpha)=\mathrm{v}(\beta)$ and, for any $A, \mathrm{v}(\sim A(\alpha))=\mathrm{v}(\sim A(\beta))$. 
Obviously (1)-(5) agree with the clauses S1.1-4 that define $v$ in the pHLsemantics.

In order to prove that $\mathrm{M}$ verifies $\Gamma$ and falsifies $A$, we show that, for all $C \in \mathcal{W}:$

$(*) \quad$ if $C \in \Delta$, then $\mathrm{v}_{\mathrm{M}}(C)=1$.

This proceeds by induction on the complexity of $C$. For the basis, we show that $(*)$ holds for all primitive wffs. This is obvious for members of $\mathcal{S}$ in view of (2) and $\mathrm{S} 2.2$.

Consider the primitive predicative wff $\pi \alpha_{1} \ldots \alpha_{n}$. If $\pi \alpha_{1} \ldots \alpha_{n} \in \Delta$, then $\mathrm{v}_{\mathrm{M}}\left(\pi \alpha_{1} \ldots \alpha_{n}\right)=1$ in view of $(3),(4)$ and S2.3. Hence $\left(^{*}\right)$ holds for primitive predicative expressions.

Consider, finally, wffs of the form $\alpha=\beta$. If $\alpha=\beta \in \Delta$, then by the definition of $\mathrm{D}$, there is a $\gamma \in \mathrm{D}$ such that $\alpha=\gamma, \beta=\gamma \in \Delta$; hence $\mathrm{v}(\alpha)=\mathrm{v}(\beta)=\gamma$ and $\mathrm{v}_{\mathrm{M}}(\alpha=\beta)=1$.

We now proceed to the induction step. All steps are standard except for negation and the universal quantifier. If $\sim C \in \Delta$, then either $C \in \Delta$ or $C \notin \Delta$. If $C \in \Delta$ then $\vee(\sim C)=1$ (by (5)); if $C \notin \Delta$, then $\vee_{\mathrm{M}}(C)=0$ by the induction hypothesis. In view of $\mathrm{S} 2.6$, either case implies that $\mathrm{v}_{\mathrm{M}}(\sim C)=1$. If $(\forall \alpha) C(\alpha) \in \Delta$, then $\sim \neg C(\beta) \in \Delta$ for all $\beta \in \mathcal{C}$ and hence $\mathrm{v}_{\mathrm{M}}(\sim \neg C(\beta))=$ 1 for all $\beta \in \mathcal{C}$; in view of $(6), \operatorname{v}_{M}(\beta)=1$ for all $\beta \in \mathcal{V}$. Hence, by $\mathrm{S} 2.11$, $\mathrm{v}_{\mathrm{M}}((\forall \alpha) C(\alpha))=1$.

As $\Delta$ is maximally non-trivial and $\mathrm{v}_{\mathrm{M}}(C)=1$ for all $C \in \Delta$, it follows immediately that $\Delta=\left\{C \mid \mathrm{v}_{\mathrm{M}}(C)=1\right\}$ and hence that $\mathrm{v}_{\mathrm{M}}(A)=0$. but $\mathrm{v}_{\mathrm{M}}(B)=1$ for all $B \in \Gamma$. Hence $\Gamma \not \neq_{\mathrm{pHL}} A$. $\square$.

\subsection{HL.}

HL has two purposes. (i) Whenever $\sim \neg A$ is pHL-derivable and $\neg A$ is not pHL-derivable from $\Gamma$, then $A$ is HL-derivable. (ii) If both $\sim \neg A$ and $\neg A$ are pHL-derivable, then $\neg A$ is HL-derivable but $A$ is not. ${ }^{8}$

\subsubsection{Proof Theory of HL.}

Where $\sharp A$ is a formula in which the variables $\alpha_{1}, \ldots, \alpha_{m}(m \geq 0)$ occur free, let $\exists \sharp A$ be $\left(\exists \alpha_{1}\right) \ldots\left(\exists \alpha_{m}\right) \sharp A$. Let $\mathrm{DEK}\left\{A_{1}, \ldots, A_{n}\right\}$ refer to $\exists \sharp A_{1} \vee \ldots \vee \exists \sharp A_{n}$, a disjunction of (where necessary) existentially quantified contradictions. $A_{1}, \ldots, A_{n}$ are the factors of $\operatorname{DEK}\left\{A_{1}, \ldots, A_{n}\right\}$.

\footnotetext{
${ }^{8}$ There are different strategies to construct an adaptive logic. The strategy followed here is the same as the strategy on which ACLuN1 is based, namely reliability. See [4].
} 
Remark that $\operatorname{DEK}(\Sigma \cup\{P x\})$ is pHL-equivalent to $\operatorname{DEK}(\Sigma \cup\{P y\})$ and pHL-derivable from $\operatorname{DEK}(\Sigma \cup\{P a\})$.

Obviously, for any $\mathcal{N}$-normal model $\mathrm{M}, \mathrm{v}_{\mathrm{M}}(\exists \sharp A)=0$.

Theorem 6 If there are $C_{1}, \ldots, C_{n} \in \mathcal{F}(0 \leq n)$ such that $\Gamma \models_{\text {pHL }}$ $\mathrm{DEK}\left\{C_{1}, \ldots, C_{n}\right\} \vee A$, then $\Gamma \mid=\mathbf{C L} A$.

Proof. It follows from the antecedent that any $\mathrm{pHL}$-model $\mathrm{M}$ verifies $\exists \sharp C_{1}$ or ... or $\exists \sharp C_{n}$ or $A$. Any CL-model is equivalent to a $\mathcal{N}$-normal model. But these models all falsify any $\exists \sharp C_{i}$. Hence any $\mathbf{C L}$-model verifies $A$.

Define the set $\operatorname{bsf}(A)$ of (open and closed) basic subformulas of $A$ as follows:

(i) if $A$ is a primitive (open or closed) formula, then $\operatorname{bsf}(A)=\{A\}$,

(ii) $\operatorname{bsf}(\neg B)=\operatorname{bsf}(B)$,

(iii) $\operatorname{bsf}(\sim B)=\{\sim B\} \cup \operatorname{bsf}(B)$,

(iv) $\operatorname{bsf}(B \vee C)=\operatorname{bsf}(B \supset C)=\operatorname{bsf}(B \& C)=\operatorname{bsf}(B \equiv C)=\operatorname{bsf}(B) \cup \operatorname{bsf}(C)$,

(v) $\operatorname{bsf}((\forall \alpha) A(\alpha))=\operatorname{bsf}(\sim \neg A(\alpha))$,

(vi) $\operatorname{bsf}((\exists \alpha) A(\alpha))=\operatorname{bsf}(A(\alpha))$.

$\operatorname{bsf}(A)$ is finite for any $A$.

Lemma 1 For any pHL-model $\mathrm{M}$, if there is no $B$ such that $\sim B \in \operatorname{bsf}(A)$ and $\mathrm{v}_{\mathrm{M}}(\exists \sharp B)=1$, then there is a $\mathcal{N}$-normal model $\mathrm{M}$ ' such that $\mathrm{v}_{\mathrm{M}}(A)=$ $\mathrm{v}_{\mathrm{M}^{\prime}}(A)$.

Proof. Suppose the antecedent is true for some $M$. Let $\mathrm{M}^{\prime}$ be obtained from $\mathrm{M}$ by putting $\mathrm{v}(\sim B)=0$ for all $B(\mathrm{~m})$. We proceed by an induction on the complexity of $A$ (the number of quantifiers and connectives that occur in $A$ ). If the complexity of $A$ is 0 , then $\mathrm{v}_{\mathrm{M}^{\prime}}(A)=\mathrm{v}_{\mathrm{M}}(A)$. Supposing that $\mathrm{v}_{\mathrm{M}^{\prime}}(A)=\mathrm{v}_{\mathrm{M}}(A)$ for all $A$ with complexity less than $n$, I show that $\mathrm{v}_{\mathrm{M}^{\prime}}(A)=\mathrm{v}_{\mathrm{M}}(A)$ for all $A$ with complexity $n$. Of the eight cases to be considered, five are obvious, viz. the ones where $A$ is either $\neg B$ or $B \supset C$ or $B \& C$ or $B \vee C$ or $B \equiv C$.

Case 6: $A$ is $\sim B$. If $\mathrm{v}_{\mathrm{M}^{\prime}}(B)=\mathrm{v}_{\mathrm{M}}(B)=0$, then $\mathrm{v}_{\mathrm{M}^{\prime}}(\sim B)=\mathrm{v}_{\mathrm{M}}(\sim B)=1$ by S2.6. Suppose that $\mathrm{v}_{\mathrm{M}^{\prime}}(B)=\mathrm{v}_{\mathrm{M}}(B)=1$. Then $\mathrm{v}_{\mathrm{M}^{\prime}}(\sim B)=0$ (as $\mathrm{M}^{\prime}$ is a $\mathcal{N}$-normal model). But also $\mathrm{v}_{\mathrm{M}}(\sim B)=0$, for otherwise, in view of S2.6, $\mathrm{v}(\sim B)=\mathrm{v}_{\mathrm{M}}(\sharp B)=\mathrm{v}_{\mathrm{M}}(\exists \sharp B)=1$, which contradicts the main supposition.

Case 7: $A$ is $(\exists \alpha) B(\alpha)$. Suppose first that $\mathrm{v}_{\mathrm{M}}(A)=1$. Then $\mathrm{v}_{\mathrm{M}}(B(\beta))=$ 1 for at least one $\beta \in \mathcal{C} \cup \mathcal{V}$. Hence, by the induction hypothesis, $\mathrm{v}_{\mathbf{M}^{\prime}}(B(\beta))=$ 1 for at least one $\beta \in \mathcal{C} \cup \mathcal{V}$. But then $\vee_{\mathrm{M}^{\prime}}((\exists \alpha) B(\alpha))=1$. Suppose next that 
$\mathrm{v}_{\mathrm{M}}(A)=0$. Then $\mathrm{v}_{\mathrm{M}}(B(\beta))=0$ for all $\beta \in \mathcal{C} \cup \mathcal{V}$. Hence, by the induction hypothesis, $\mathrm{v}_{\mathrm{M}^{\prime}}(B(\beta))=0$ for all $\beta \in \mathcal{C} \cup \mathcal{V}$. But then $\mathrm{v}_{\mathrm{M}^{\prime}}((\exists \alpha) B(\alpha))=0$.

Case 8: $A$ is $(\forall \alpha) B(\alpha)$. Suppose first that $\mathrm{v}_{\mathrm{M}}(A)=1$. Then $\mathrm{v}_{\mathrm{M}}(\sim \neg B(\beta))$ $=1$ for all $\beta \in \mathcal{C} \cup \mathcal{V}(\mathrm{k})$. As $\operatorname{bsf}(\sim \neg B(\alpha))=\operatorname{bsf}(A)$ (by $(\mathrm{v})), \sim \neg B(\alpha) \in$ $\operatorname{bsf}(A)$. But then $\mathrm{v}_{\mathrm{M}}(\exists \sharp \neg B(\alpha))=0$ and hence $\mathrm{v}_{\mathrm{M}}(\neg B(\beta))=0$ for all $\beta \in$ $\mathcal{C} \cup \mathcal{V}$ (in view of $(\mathrm{k}))$. But then, by the induction hypothesis, $\mathrm{v}_{\mathrm{M}^{\prime}}(\neg B(\beta))=$ 0 for all $\beta \in \mathcal{O}$, and hence $\mathrm{v}_{\mathrm{M}^{\prime}}(\sim \neg B(\beta))=1$ for all $\beta \in \mathcal{C} \cup \mathcal{V}$. But then $\mathrm{v}_{\mathrm{M}^{\prime}}((\forall \alpha) B(\alpha))=1$. Suppose next that $\mathrm{v}_{\mathrm{M}}(A)=0$. Then there is a $\beta \in \mathcal{C} \cup \mathcal{V}$ such that $\mathrm{v}_{\mathrm{M}}(\sim \neg B(\beta))=0$. Then $\mathrm{v}_{\mathrm{M}}(\neg B(\beta))=1$ for at least one $\beta \in \mathcal{C} \cup \mathcal{V}$, and hence, by the induction hypothesis, $\mathbf{v}_{\mathbf{M}^{\prime}}(\neg B(\beta))=1$ for the same $\beta \in \mathcal{C} \cup \mathcal{V}$. But then in view of $(\mathrm{m})$ and $\mathrm{S} 2.6, \mathrm{v}_{\mathrm{M}^{\prime}}(\sim \neg B(\beta))=0$ for at least one $\beta \in \mathcal{C} \cup \mathcal{V}$, and hence $\mathrm{v}_{\mathrm{M}^{\prime}}((\forall \alpha) B(\alpha))=0$. $\square$.

Theorem 7 If $\models_{\mathrm{CL}} A$, then for some $C_{1}, \ldots, C_{n} \in \mathcal{F}(0 \leq n),\left.\right|_{\mathrm{pHL}}$ $\mathrm{DEK}\left\{C_{1}, \ldots, C_{n}\right\} \vee A$.

Proof. Let $\models_{\mathbf{C L}} A$. Hence $\mathrm{v}_{\mathrm{M}}(A)=1$ for all $\mathcal{N}$-normal models $\mathrm{M}$ of $\Gamma$. As $\operatorname{bsf}(A)$ is finite, $\operatorname{DEK}\{B \mid \sim B \in \operatorname{bsf}(A)\} \vee A$ is a wff, which is easily shown to be $\mathbf{p H L}$-valid. Consider indeed a $\mathbf{p H L}$-model $\mathrm{M}$. If, for some $\sim B \in \operatorname{bsf}(A) \vee_{\mathrm{M}}(\exists \sharp B)=1$, then $\mathrm{v}_{\mathrm{M}}(\operatorname{DEK}\{B \mid \sim B \in \operatorname{bsf}(A)\})=1$. If, for no $\sim B \in \operatorname{bsf}(A), \mathrm{v}_{\mathrm{M}}(\exists \sharp B)=1$, then $\mathrm{v}_{\mathrm{M}}(A)=1$ by Lemma 1 .

Some formulas in the second column HL-proofs are derived conditionally, and the lines in which they occur, have to be marked when a condition is overruled. Formulas in the second element of marked lines do not belong to the consequence set. Conditions are all of one kind: some $C_{1}, \ldots, C_{n}$ have to behave $\sim$-consistently.

The idea of the proof theory of $\mathbf{H L}$ is that we apply all rules derivable in pHL unconditionally, whereas other rules derivable in CL are applied on condition that certain formulas are reliable with respect to their consistent behaviour. To keep the matter algorithmic, the consistent behaviour of a formula will be determined by the stage of the proof instead of by the abstract notion of derivability. As a result, formulas derived at some stage of proof, will not be finally derivable, because the line in which they occur will be marked at a later stage. Of course each set of premises must (and will) have a unique set of final HL-consequences.

As shown in the example above, HL-proofs are written in a special format according to which each line of a proof consist of five elements:

(i) a line number,

(ii) a premise, a theorem or a derived formula,

(iii) the line numbers of the wffs from which (ii) is derived, 
(iv) the rule of inference that justifies the derivation, and

(v) the formulas on the consistent behaviour of which we rely in order for (ii) to be derivable by (iv) from the formulas of the lines enumerated in (iii).

Definition. A occurs unconditionally at some line of a proof iff the fifth element of that line is empty.

Definition. A behaves consistently at a stage of a proof iff $\sharp A$ does not occur unconditionally in the proof at that stage.

Definition. The consistent behaviour of $A_{1}$ is connected to the consistent behaviour of $A_{2}, \ldots, A_{n}$ at a stage of a proof iff $\operatorname{DEK}\left\{A_{1}, \ldots, A_{n}\right\}$ occurs unconditionally in the proof at that stage whereas $\operatorname{DEK}\left\{A_{2}, \ldots, A_{n}\right\}$ does not occur unconditionally in it.

Definition. $A$ is reliable at a stage of a proof iff $A$ behaves consistently at that stage and its consistent behaviour is not connected to the consistent behaviour of other formulas.

Given these definitions, proofs in HL are governed by an unconditional rule, a conditional rule and a marking rule. The application of a rule to a proof at a stage produces the next stage.

RU If $\vdash_{\mathrm{pHL}}\left(A_{1} \& \ldots \& A_{n}\right) \supset B$, and $A_{1}, \ldots, A_{n}$ occur in the proof, then add $B$ to it. The fifth element of the new line is the union of the fifth elements of the lines mentioned in its third element.

$\mathrm{RC} \quad$ If $\vdash_{\mathrm{pHL}} \operatorname{DEK}\left\{C_{1}, \ldots, C_{m}\right\} \vee\left(\left(A_{1} \& \ldots \& A_{n}\right) \supset B\right)$, and $A_{1}, \ldots$, $A_{n}$ occur in the proof, then add $B$ to it, provided that each factor of $\operatorname{DEK}\left\{C_{1}, \ldots, C_{m}\right\}$ is reliable at that stage. The fifth element of the new line is the union of $\left\{C_{1}, \ldots, C_{m}\right\}$ and of the fifth elements of the lines mentioned in its third element.

$\mathrm{RM}$ If $C$ is not (any more) reliable, then mark all lines the fifth element of which contains $C$, by writing " $\uparrow$ " before the line number. A marked line does not belong to the proof at that stage. $^{9}$

At any stage of the proof, it is obligatory to apply RM and permitted to apply RU and RC. If the fifth element of a line is empty, the formula in its second element is $\mathbf{p H L}$-derivable from the premises and cannot be marked later. If the fifth element is not empty, its formula is provisionally derived. Unless it can also be derived at a line the fifth element of which

\footnotetext{
${ }^{9}$ At every stage of a proof, previously introduced marks are omitted, and marks are introduced according to the reliability at the new stage.
} 
is empty, it is not a pHL-consequence. The unconditional occurrence of DEK-formulas in the proof determines whether some formulas are reliable, and hence which applications of RC are permitted in view of pHL-formulas of the form $\operatorname{DEK}\left\{C_{1}, \ldots, C_{m}\right\} \vee\left(\left(A_{1} \& \ldots \& A_{n}\right) \supset B\right)$. As usual proofs may be sped up by derived rules. Of course all positive rules of CL are valid unconditionally. RAA, Reductio ad absurdum in the case of " $\neg$ ", $(A \supset B$, $A \supset \neg B / \neg A$ ) cannot be applied if the fifth element of the premise lines is not empty. ${ }^{10}$ Obviously $\mathbf{R} \forall$ (to derive $\vdash A \supset(\forall \alpha) B(\alpha)$ from $\vdash A \supset \sim \neg B(\beta)$, provided $\beta$ does not occur in either $A$ or $B(\alpha)$ ) cannot be applied if $\sim \neg B(\beta)$ is the second element of a line the fifth element of which contains a formula in which $\beta$ occurs. I list some specific HL-rules. The mentioned sets refer to the fifth element of the line.

- Negation Rules:

$\mathrm{NR}: \neg A, \Sigma / \sim A, \Sigma$

CNR: $\sim A, \Sigma / \neg A, \Sigma \cup\{A\}$

UI: Instance of a Universal Quantification:

$(\forall \alpha) A(\alpha), \Sigma / \sim \neg A(\beta), \Sigma$

DN: Double Negation:

$A, \Sigma / \sim \neg A, \Sigma$

$\neg \sim A, \Sigma / A, \Sigma$

CDN: Conditional Double Negation:

$\sim \neg A, \Sigma / A, \Sigma \cup\{\neg A\}$

$\sim \sim A, \Sigma / A, \Sigma \cup\{\sim A\}$

$A, \Sigma / \neg \sim A, \Sigma \cup\{A\}$

HL has a dynamic proof procedure: a wff may be derived at some stage of a proof, while the line in which it occurs may be marked at a later stage of the proof; and a formula that is not reliable at some stage (which results in the marking of all lines with this formula in their fifth element), may become reliable at a later stage. Therefore we need to distinguish between provisional and final consequences.

Definition. $A$ is finally derived at some line in an HL-proof iff (i) it is the second element of that line and (ii) where $\left\{C_{1}, \ldots, C_{n}\right\} \quad(n \geq 0)$ is the fifth element of the line, any extension of the proof can be further extended in such way that it contains a line that has $A$ as its second element and $\left\{C_{1}, \ldots, C_{n}\right\}$ as its fifth element.

\footnotetext{
${ }^{10}$ The reason is obvious. Suppose $A \supset B$ is derived in a line of which the fifth element is $C$. This means that $\sharp C \vee(A \supset B)$ (and not $A \supset B$ ) is pHL-derived (see Lemma 2, below); it is clear that the simultanous occurrence of $\sharp C \vee(A \supset B)$ and $A \supset \neg B$ does not mean that the occurrence of $A$ leads to triviality.
} 
Sometimes a $C_{i}$ is unreliable in an extension of the proof (in that its consistent behaviour becomes connected to that of other formulas). However, if any extension can be further extended in such way that all $C_{i}$ are reliable, then $A$ is derived from $\Gamma$ on the condition $\left\{C_{1}, \ldots, C_{n}\right\}$ in an absolute sense (which is studied further below).

Definition. $\Gamma \vdash_{\mathbf{H L}} A$ ( $A$ is finally $\mathbf{H L}$-derivable from $\Gamma$ ), iff $A$ is finally derived at some line in a $\mathbf{H L}$-proof from $\Gamma$.

Definition. (The HL-consequence set of $\Gamma$ ) $C n_{\mathbf{H L}}(\Gamma)=\left\{A \mid \Gamma \vdash_{\mathbf{H L}} A\right\}$.

pHL is not decidable. We lack a positive test for HL-derivability. Of course, some fragments of $\mathbf{H L}$ are decidable.

Yet, it is possible to prove that $C n_{\mathbf{H L}}(\Gamma)$ may be characterized without referring to the dynamics of the proofs. The characterization refers to $\mathbf{p H L}$ only. The central point is that it depends only on pHL-derivability (which is monotonic) whether a wff is reliable in an intelligent extension of the proof.

Lemma 2 If in an HL-proof from $\Gamma, A$ occurs as the second element and $\left\{C_{1}, \ldots, C_{m}\right\}(0 \leq m)$ occurs as the fifth element of a line, then $\Gamma \vdash_{\mathrm{pHL}}$ $A \vee \operatorname{DEK}\left\{C_{1}, \ldots, C_{m}\right\} .^{11}$

In view of this lemma, we can introduce the following derivation rule.

DEK: $A, \Sigma / A \vee \operatorname{DEK}(\Sigma)$

DEFInItion. A DEK-consequence of $\Gamma$ is a DEK-formula which is pHLderivable from $\Gamma$.

Definition. $\operatorname{DEK}(\Delta)$ is a minimal DEK-consequence of $\Gamma$ iff it is a DEKconsequence of $\Gamma$, and for no $\Phi \subset \Delta, \operatorname{DEK}(\Phi)$ is a DEK-consequence of $\Gamma$.

Theorem $8 \Gamma \vdash_{\text {HL }} A$, iff there are $C_{1}, \ldots, C_{m} \in \mathcal{F}(0 \leq m)$ such that $\Gamma \vdash_{\mathrm{pHL}} A \vee \mathrm{DEK}\left\{C_{1}, \ldots, C_{m}\right\}$, and none of $C_{1}, \ldots, C_{m}$ is a factor of a minimal DEK-consequence of $\Gamma{ }^{12}$

It follows from Theorem 8 that whenever $A$ occurs as the second element of a line of a HL-proof in which $C_{1}, \ldots, C_{m}$ is the fifth element, a new line can be added with $A \vee \operatorname{DEK}\left\{C_{1}, \ldots, C_{m}\right\}$ as second element and an empty fifth element, and vice versa.

The following Theorem expresses an important feature of HL:

\footnotetext{
${ }^{11}$ The proof of Lemma 2 is completely analogous to the proof of Lemma 1 in [1] and Lemma 4.2 in [4].

${ }^{12}$ The proof of Theorem 8 is completely analogous to the proof of Theorem 4.3 in [4].
} 
Theorem 9 If $\Gamma \vdash_{\text {HL }} A$, then it is possible to extend any proof from $\Gamma$ into a proof in which $A$ is finally derived from $\Gamma .{ }^{13}$

I now give a derivable marking rule in $\mathbf{H L}$.

MR1: If $A$ (resp. $\neg A$ ) is conditionally derived at line $i$ of a $\mathbf{H L}$ proof from $\Gamma$, while $\neg A$ (resp. $A$ ) is derived unconditionally at any line of the proof, then mark line $i$.

Theorem 10 The marking rule MR1 is derivable in HL.

Proof. By assumption, $A$ occurs in a HL-proof from $\Gamma$ as the second element of a line (i) the fifth element of which is $C_{1}, \ldots, C_{n}(1 \leq n)$. By Lemma 2, $\operatorname{DEK}\left\{C_{1}, \ldots, C_{n}\right\} \vee A$ is a $\mathbf{p H L}$-consequence of $\Gamma$, and hence can be derived unconditionally in the $\mathbf{H L}$-proof. Suppose $\neg A$ occurs at line $(\mathrm{j})$ with an empty fifth element. In view of the PHL-theorem $A \vee \neg A$, also $\operatorname{DEK}\left\{C_{1}, \ldots, C_{n}\right\}$ is unconditionally derivable. But then at least one of its factors is unreliable and hence all lines with each of the formulas $C_{1}, \ldots$ or $C_{n}$ in their fifth element have to be marked, in view of RM. The proof is completely analogous if we replace $A$ by $\neg A$ and vice versa. $\square$.

\subsubsection{Semantics of $\mathrm{HL}$}

The HL-semantics is obtained from the pHL-semantics by defining, for each $\Gamma$, a subset of the pHL-models of $\Gamma$. The idea is that any $\Gamma$ defines a set of (semantically) unreliable formulas, and that the HL-models of $\Gamma$ are those pHL-models of $\Gamma$ in which only unreliable formulas behave inconsistently.

Definition. $A$ is HL-unreliable with respect to $\Gamma$ iff $A$ is a factor of a minimal DEK-consequence of $\Gamma . \mathcal{U}(\Gamma)$ is the set of all wffs that are HLunreliable with respect to $\Gamma$.

Definition: Where $\mathrm{M}$ is a pHL-model, $\mathrm{ab}(\mathrm{M})=\left\{A \mid \mathrm{v}_{\mathrm{M}}(\sharp A)=1\right\}$.

Definition: $M$ is a HL-model of $\Gamma$ iff it is a pHL-model of $\Gamma$ and $a b(M) \subseteq$ $\mathcal{U}(\Gamma)$.

Definition: $\Gamma \mid=\mathbf{H L} A$ iff $A$ is true in all $\mathbf{H L}$-models of $\Gamma$.

\subsubsection{Metatheory of HL.}

Theorem 11 If $\Gamma \vdash_{\text {HL }} A$, then $\Gamma \models_{\text {HL }} A$.

Proof. Let $\Gamma \vdash_{\text {HL }} A$. By Theorem 8 , there are $C_{1}, \ldots, C_{m}(m \geq 0)$ such that $\Gamma \vdash_{\mathrm{pHL}} A \vee \operatorname{DEK}\left\{C_{1}, \ldots, C_{m}\right\}$, and $C_{1}, \ldots, C_{m} \notin \mathcal{U}(\Gamma)$. If $\Gamma \mid{ }_{\mathrm{pHL}} A$, then

\footnotetext{
${ }^{13}$ The proof of Theorem 9 is completely analogous to the proof of Theorem 4.4 in [4].
} 
$\Gamma \models \mathbf{H L} A$ (all HL-models are $\mathbf{p H L}$-models). If there are $C_{1}, \ldots, C_{m}(m \geq 1)$ such that $\Gamma \vdash_{\mathrm{pHL}} A \vee \operatorname{DEK}\left\{C_{1}, \ldots, C_{m}\right\}$, and $C_{1}, \ldots, C_{m} \notin \mathcal{U}(\Gamma)$, then, for all HL-models $\mathrm{M}$ of $\Gamma, \mathrm{v}_{\mathrm{M}}\left(A \vee \operatorname{DEK}\left\{C_{1}, \ldots, C_{m}\right\}\right)=1$, and $\mathrm{v}_{\mathrm{M}}\left(\mathrm{DEK}\left\{C_{1}, \ldots, C_{m}\right\}\right)$ $=0$, and hence $\mathrm{v}_{\mathrm{M}}(A)=1$. $\square$.

Theorem 12 If $\Gamma=_{\text {HL }} A$, then $\Gamma \vdash_{\text {HL }} A$.

Proof. Suppose that $\Gamma \models_{\mathbf{H L}} A$ and $\Gamma \nvdash_{\mathbf{H L}} A$. Let the sequence $B_{1}, B_{2}, \ldots$ be defined as in the proof of Theorem 5 . We define

$$
\begin{aligned}
& \Delta_{0}=C n_{\mathbf{p H L}}(\Gamma \cup\{\operatorname{DEK}\{B\} \supset A \mid B \in \mathcal{W}-\mathcal{U}(\Gamma)\}) .{ }^{14} \\
& \Delta_{i+1}=C n_{\mathbf{p H L}}\left(\Delta_{1} \cup\left\{B_{i+1}\right\}\right) \text { if } A \notin C n_{\mathbf{p H L}}\left(\Delta_{1} \cup\left\{B_{i+1}\right\}\right), \text { and } \\
& \Delta_{i+1}=\Delta_{i} \text { otherwise } \\
& \Delta=\Delta_{0} \cup \Delta_{1} \cup \ldots
\end{aligned}
$$

Each of the following is provable:

(i) $\quad \Gamma \subseteq \Delta$

(ii) $A \notin \Delta$. By the definition of $\Delta$, if $A \in \Delta$, then $A \in \Delta_{0}$. The latter however is impossible. Indeed, if $A \in \Delta_{0}$, then there are $C_{1}, \ldots, C_{m} \in$ $\mathcal{F}-\mathcal{U}(\Gamma)(m \geq 1)$ such that $\Gamma \cup\left\{\operatorname{DEK}\left\{C_{1}, \ldots, C_{m}\right\} \supset A\right\} \vdash_{\text {pHL }} A .{ }^{15}$ Hence, by the deduction theorem, $\Gamma \vdash_{\mathrm{pHL}}\left(\operatorname{DEK}\left\{C_{1}, \ldots, C_{m}\right\} \supset A\right) \supset A$; hence $\Gamma \vdash_{\mathrm{pHL}} \operatorname{DEK}\left\{C_{1}, \ldots, C_{m}\right\} \vee A$. But as $C_{1}, \ldots, C_{m} \in \mathcal{F}-\mathcal{U}(\Gamma)$, it follows by Theorem8 that $\Gamma \vdash_{\text {HL }} A$, which contradicts (the main supposition.

(iii) If $C \notin \mathcal{U}(\Gamma)$, then $\exists \sharp C \notin \Delta$. Indeed, if $C \notin \mathcal{U}(\Gamma)$, then $\sharp C \supset A \in$ $\Delta_{0}$; so if $\sharp C \in \Delta$, then $A \in \Delta$, which contradicts (ii).

(iv) $\Delta$ is deductively closed (by the definition of $\Delta$ ).

(v) $\Delta$ is maximally non-trivial (as in the proof of Theorem 5 .

As in the proof of Theorem 5, a $\mathbf{p H L}$-model $\mathrm{M}$ is a HL-model is defined from $\Delta$. In view of (i) and (ii), all members of $\Gamma$ are true in $\mathrm{M}$ and $A$ is false in M. In view of (iii), $\mathrm{M}$ is a $\mathbf{H L}$-model of $\Gamma$. Hence $\Gamma \nvdash \mathbf{H L} A$. $\square$.

\section{HL1.}

The proof theory and the semantics of $\mathbf{H L} \mathbf{1}$ are obtained by adding the CL-clauses concerning the universal quantifier to the proof theory and the

\footnotetext{
${ }^{14}$ It is in view of the fact that $\mathcal{U}(\Gamma)$ is definied by means of the semantical notion minimal DEK-consequences, that the construction of $\Delta$ captures the dynamic proof procedure. Remember that in Theorem $8 \Gamma \vdash_{\mathbf{H L}} A$ is defined without referring to the dynamics of HL-proofs.

${ }^{15} \mathrm{In}$ view of the fact that any pHL-proof is finite, and of $\{A \supset B, C \supset B\} \vdash_{\mathrm{pHL}}$ $(A \vee C) \supset B$.
} 
semantics of $\mathbf{H L}$. We write the CL-quantifier as " $\forall$ " ", and the HL-quantifier as " $\forall$ ". The metatheory of $\mathbf{p H L 1}$ and $\mathbf{H L} \mathbf{1}$ is completely analogous to those of pHL and HL. An example of a HL1-proof will show the usefulness of HL1. ${ }^{16}$

(1) $(\forall x)(B x \supset F x) \quad$ - $\quad$ PREM-

(2) $\left(\forall^{0} x\right)(P x \supset B x)$ - PREM-

(3) $\left(\forall^{0} x\right)(P x \supset \neg F x)$ - $\quad$ PREM-

(4) $\mathrm{Pa} \quad$ - PREM-

(5) $\mathrm{Bb} \quad-\quad$ PREM-

(6) $\sim \neg(B a \supset F a) \quad 1 \quad$ UI $\quad-$

(7) $\mathrm{Ba} \supset \mathrm{Fa} \quad 6 \quad \mathrm{CDN} \neg(B a \supset F a)$

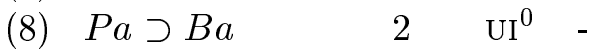

(9) $\mathrm{Ba} \quad 4,8 \mathrm{MP} \quad-$

†(10) $\mathrm{Fa} \quad 7,9$ MP $\neg(B a \supset F a)$

(11) $P a \supset \neg F a \quad 3 \quad$ UI $^{0} \quad-$

(12) $\neg F a \quad 4,11 \mathrm{MP} \quad-$

(13) $\sim \neg(B b \supset F b) \quad 1 \quad$ UI -

(14) $\mathrm{Bb} \supset \mathrm{Fb} \quad 13 \quad \mathrm{CDN} \neg(B b \supset F b)$

(15) $\mathrm{Fb} \quad 5,14 \mathrm{MP} \quad \neg(B b \supset F b)$

In view of MR1 line (10) has to be marked, and hence $\neg F a$ is finally derived while $\mathrm{Fa}$ is not. ${ }^{17}$ As the condition in line (15) is not overruled (it is not possible to derive $\sharp \neg(B b \supset F b)), F b$ is finally derived.

If we construct now a proof from the premises $(\forall x)(P x \supset \neg F x)$, $\left(\forall^{0} x\right)(M x \supset P x),\left(\forall^{0} x\right)(M x \supset F x), M c$ and $P a$, we can derive $F c$ and $\neg F a$ (in a completely analogous way as in the former proof).

Suppose now we want to make one proof from these two proofs. Then we meet a problem in that we have both $\left(\forall^{0} x\right)(P x \supset \neg F x)$ and $(\forall x)(P x \supset$ $\neg F x)$. A UQF cannot be classical and exceptional at once. If we make it a classical UQF, then we can derive an inconsistency that causes triviality from the premises: both $F c$ and $\neg F c$ are derivable unconditionally. Hence, it has to be considered as a UQF that might have exceptions; but if we make it an exceptional UQF, $\neg F a$ is only derivable on unreliable conditions, and hence neither $\neg F a$ nor $F a$ are finally derivable.

\footnotetext{
${ }^{16}$ In the proofs given as example in Sections 3 and 4 , you can read " $B$ " as "is a bird", " $P$ " as "is a penguin", " $M$ " as "is a motorized penguin", and " $F "$ as "can fly".

${ }^{17}$ It is easily seen that $\sharp \neg(B a \supset F a)$ can be derived unconditionally. Once this is done, line (7) has to be marked, in view of RM.
} 
This example shows that the HL1-formalization of rules allows us to derive finally more wanted consequences than the HL-formalization. But some wanted consequences are not finally derivable, especially if the context of application extends in such a way that general statements without exceptions become general statements that might have exceptions. In HL2 however only the unwanted consequences will not be finally derivable.

\section{HL2.}

HL2 is obtained by introducing a preference ordering on the exceptional UQFs of HL1. Instead of one set of exceptional UQFs, there is a set of UQFs of preference 1 (the highest preference), ..., and a set UQFs of preference $n$ (the lowest preference). With UQFs of preference $n$ correspond instances of preference $n$ (notation: $\sim^{n} \neg A$ ). ${ }^{18}$ If $i<j$, the relation between a HL2-UQF of preference $i$ and a HL2-UQF of preference $j$, is the same as the relation between the corresponding classical HL1-UQF and the corresponding exceptional HL1-UQF. An immediate result is that, in case of a contradiction between (a formula derived from) an instance of preference $i$ and (a formula derived from) an instance of preference $j$, it will follow from the occurrence of the former that the latter is not finally derivable.

I am not dealing here with the question which UQFs have to be related to a higher or lower preference. ${ }^{19}$ In specific contexts however, preferences can often be ascribed without problems. In the example of Section 3, for instance, the premises will contain the following UQFs: $\left(\forall^{0} x\right)(P x \supset B x)$ and $\left(\forall^{0} x\right)(M x \supset P x),\left(\forall^{1} x\right)(M x \supset F x),\left(\forall^{2} x\right)(P x \supset \neg F x)$ and $\left(\forall^{3} x\right)(B x \supset$ $F x$ ). In this section it will become clear that $F c, \neg \mathrm{Fa}$ and $F b$ are finally derivable from these premises.

\subsection{The Underlying Paraconsistent Logic pHL2.}

\subsubsection{Proof Theory of pHL2.}

The underlying paraconsistent logic of HL2, is pHL2, which is obtained from pHL1 by introducing preferences in the language scheme: there are $n$ exceptional universal quantifiers: $\forall^{1}, \ldots, \forall^{n}$, and $n$ paraconsistent negations: $\sim^{1}, \ldots, \sim^{n}$. The axiom scheme is obtained by replacing in pHL1 $\mathbf{A} \forall, \mathbf{R} \forall$ and $\mathbf{A} \sim$ by: (for all $n \geq 1)$

\footnotetext{
${ }^{18} \sim^{n} \neg A$ can be read as "we give preference $n$ to the fact that there is no reason to reject $A$.

${ }^{19}$ For an extended study on preferences, I refer to, e.g., [7].
} 
$\mathbf{A} \forall^{n}: \quad\left(\forall^{n} \alpha\right) A(\alpha) \supset \sim^{n} \neg A(\beta)$

$\mathbf{R} \forall^{n}: \quad$ To derive $\vdash A \supset\left(\forall^{n} \alpha\right) B(\alpha)$ from $A \supset \sim^{n} \neg B(\beta)$, provided $\beta$ does not occur in either $A$ or $B(\alpha)$

$\mathbf{A} \sim^{n}: \quad A \vee \sim^{n} A$

The Fitch-style rule Universal Instantiation from a UQF with preference $n(\geq 1)$ is indicated as "UI"

Obviously, the pHL2-consequences are the pHL1-consequences to which the preferences are added where necessary.

\subsubsection{Semantics of pHL2.}

The semantics of pHL2 is obtained from the pHL1-semantics by replacing $\mathcal{N}$ by $n$ sets $\mathcal{N}^{n}=\left\{\sim^{n} A \mid A \in \mathcal{F}\right\}$. In the definition of the assignment function $\mathrm{S} 1.4$ is replaced by:

S1.4. $\quad$ v $: \mathcal{N}^{n} \rightarrow\{0,1\}$, for each $n \geq 1$.

The valuation function is defined by replacing in the pHL1-clauses S2.6 and S2.11 and $\forall$ by $\sim^{n}$ and $\forall^{n}$ for each $n \geq 1$.

\subsubsection{Metatheory of pHL2.}

If we take in account the above mentioned differences between pHL1 and pHL2, the metatheory of pHL2 is completely analogous to the metatheory of pHL1.

\subsection{HL2.}

HL2 has three purposes. (i) Whenever $\sim^{n} \neg A$ is pHL2-derivable and $\neg A$ is not pHL2-derivable from $\Gamma$, then $A$ is HL2-derivable from $\Gamma$. (ii) If both $\sim^{n} \neg A$ and $\neg A$ are pHL2-derivable, then $\neg A$ is HL2-derivable, but $A$ is not. (iii) If both $\operatorname{DEK}(\Sigma) \vee A$ and $\operatorname{DEK}(\Pi) \vee \neg A$ are pHL2-derivable, then the conditional preferences of the factors of $\operatorname{DEK}(\Sigma)$ and $\operatorname{DEK}(\Pi)$ decide whether $A$ or $\neg A$ is finally derivable.

\subsubsection{Proof Theory of HL2.}

Let $\exists \sharp^{n} A$ stand for $\exists\left(A \& \sim^{n} A\right)$. Let DEK $\left\{A_{1}^{i}, \ldots, A_{n}^{j}\right\}$ refer to $\exists \sharp^{i} A_{i} \vee \ldots \vee$ $\exists \sharp^{j} A_{n}(i, j \geq 1)$, a disjunction of (where necessary) existentially quantified contradictions (confer Section 2.2.1). The format of HL2-proofs is the same as for HL and HL1. 
Definition: The $\sim^{i}$-consistent behaviour of a formula $B$ is a condition of preference $i$ iff $\sim^{i} B$ is an instance of a exceptional UQF of preference $i$. The number $i$ is the conditional preference of $B$. (Notice that a higher number corresponds to a lower conditional preference. $)^{20}$

Definition: $A$ is not reliable at a stage of a proof iff it does not behave consistently at that stage of the proof or its consistent behaviour is connected to the consistent behaviour of other formulas none of which has a lower conditional preference than $A$.

Given these HL2-definitions, the unconditional rule RU, the conditional rule $\mathbf{R C}$, and the marking rule $\mathbf{R M}$ are completely analogous as for $\mathbf{H L}$ and HL1. The following example illustrates the difference between the rule RM in HL1 and HL2. Suppose lines (i)-(k) occur in a proof: ${ }^{21}$

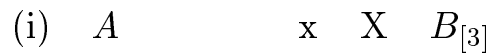

(j) $\neg A \quad$ y $\quad$ Y $\quad C_{[2]}$

(k) $\sharp^{3} B \vee \sharp^{2} C \quad \mathrm{z} \quad \mathrm{Z} \quad-$

If we drop the preferences, both $B$ and $C$ would be unreliable, and hence neither $A$ nor $\neg A$ would be finally derivable from these lines (this is the situation in HL1). But in view of the fact that the conditional preference of $C(2)$ is higher than the conditional preference of $B(3), C$ is reliable in HL2 and hence $\neg A$ is HL2-derivable from these lines.

Lemma 3 If in an HL2-proof from $\Gamma, A$ occurs as the second element and $\left\{C_{1}, \ldots, C_{m}\right\} \quad(0 \leq m)$ as the fifth element of a line, then $\Gamma \vdash_{\text {pHL2 }}$ $A \vee \operatorname{DEK}\left\{C_{1}, \ldots, C_{m}\right\} .{ }^{22}$

The definition of "intelligent extension", " $A$ is finally derived", "final derivability", "consequence set" and "minimal DEK-consequence" remain the same as for HL1 and HL. Also the derivation rule DEK is valid in HL2.

Theorem $13 \Gamma \vdash_{\text {HL2 }} A$ iff there are $C_{1}, \ldots, C_{m}(0 \leq m)$ such that $\Gamma \vdash_{\mathbf{p H L 2}}$ $A \vee \operatorname{DEK}\left\{C_{1}, \ldots, C_{m}\right\}$, and none of $C_{1}, \ldots, C_{m}$ is a factor of a minimal DEKconsequence of $\Gamma$ unless some other factor of the minimal DEK-consequence has a lower conditional preference than the considered $C_{i}$.

Proof. For the first direction, let $\Gamma \vdash_{\text {HL2 }} A$. Hence $A$ is finally derived at some line (j) of a proof from $\Gamma$. Let the fifth element of this line be $C_{1}, \ldots, C_{m}$.

\footnotetext{
${ }^{20}$ One and the same UQF never gets two different preferences. Therefore we can accept that every formula has (at maximum) one conditional preference.

${ }^{21} \mathrm{It}$ is handy to indicate the conditional preference of the formulas in the fifth column of a proof.

${ }^{22}$ The proof of Lemma 3 is completely analogous to the proofs of Lemma 1 in [1] and Lemma 4.2 in [4].
} 
Hence $\Gamma \vdash_{\mathbf{p H L} 2} A \vee \operatorname{DEK}\left\{C_{1}, \ldots, C_{m}\right\} . \Gamma \vdash_{\mathbf{p H L 2}} A \vee \operatorname{DEK}\left\{C_{1}, \ldots, C_{m}\right\}(0 \leq$ $m$ ). Suppose now that $C_{i}$ is a factor of a minimal DEK-consequence $D$ of $\Gamma$ and there is no other factor of $D$ with a lower conditional preference than $C$; then there is an extension of the proof in which $D$ occurs unconditionally; but then line ( $\mathrm{j}$ ) is marked by $\mathrm{RM}$, which contradicts the fact that $A$ is finally derived at line (j).

For the other direction, suppose that there are $C_{1}, \ldots, C_{m}(0 \leq m)$ such that $\Gamma \vdash_{\mathrm{pHL2}} A \vee \operatorname{DEK}\left\{C_{1}, \ldots, C_{m}\right\}$, and none of $C_{1}, \ldots, C_{m}$ is a factor of a minimal DEK-consequence of $\Gamma$ unless some other factor has a lower conditional preference. Then there is a an HL2-proof from $\Gamma$ in which $A$ occurs as the second element of a line the fifth element of which is $\left\{C_{1}, \ldots, C_{m}\right\}$. Moreover, every extension of the proof in which line (j) would be marked (because some $C_{i}$ is not reliable, can be further extended in such way that $C_{i}$ becomes reliable again (in view of the supposition). It follows that $A$ is finally derived at that line. Whence $\Gamma \vdash_{\text {HL2 }} A$. $\square$.

In HL and HL1 no factor of a minimal DEK-consequence is reliable. In HL2 however, those factors of a minimal DEK-consequence are reliable the conditional preference of which is higher than the conditional preference of some other factor of that minimal DEK-consequence.

The marking rule MR1 of HL and HL1 is valid in HL2. I now give the typical HL2-rule concerning marking of instances.

Definition: If $A$ occurs as the second element of a line of a proof, the line preference of $A$ is the lowest conditional preference (i.e. the highest number) of the formulas in the fifth element of that line. If the fifth element is empty, the line preference of $A$ is 0 .

MR2: If the line preference of $A$ (resp. $\neg A$ ) is lower than the line preference of $\neg A$ (resp. $A$ ) at any line of the proof, then mark line (i).

Theorem 14 MR2 is a derivable rule of HL2.

Proof. Suppose $\Sigma=\left\{B_{1}, \ldots, B_{n}\right\}(n \geq 1)$, and $\Pi=\left\{C_{1}, \ldots, C_{m}\right\}(m \geq 1)$, and there is a $B \in \Sigma$ such that the conditional preference of $B$ is lower than the conditional preference of any $C \in \Pi$. Suppose $A$ occurs as the second element of a line the fifth element of which is $\Sigma$, and $\neg A$ occurs as the second element of a line the fifth element of which is $\Pi$. In view of Lemma 3, both $A \vee \operatorname{DEK}(\Sigma)$ and $\neg A \vee \operatorname{DEK}(\Pi)$ are pHL2-derivable from the premises. Hence also $\operatorname{DEK}(\Sigma \cup \Pi)$ is derivable from the premises. In view of the supposition and the definition $B$ is not reliable at that stage of the proof. Hence the line in which $A$ was derived has to be marked in view 
of RM. The proof is completely analogous if $A$ is replaced by $\neg A$ and vice versa. $\square$.

Here is an example of a HL2-proof that illustrates the mechanism:

(1) $\left(\forall^{3} x\right)(B x \supset F x) \quad$ - $\quad$ PREM -

(2) $\left(\forall^{2} x\right)(P x \supset \neg F x)$ - PREM -

(3) $\left(\forall^{1} x\right)(M x \supset F x)$ - $\quad$ PREM -

(4) $\left(\forall^{0} x\right)(M x \supset P x)$ - $\quad$ PREM -

(5) $\left(\forall^{0} x\right)(P x \supset B x) \quad$ - $\quad$ PREM -

(6) $\mathrm{Bb}$ - PREM -

(7) $\mathrm{Pa} \quad$ - PREM -

(8) $\sim^{3} \neg(B a \supset F a) \quad 1 \quad \mathrm{UI}^{3} \quad-$

(9) $B a \supset F a \quad 8 \quad$ CDN $\neg(B a \supset F a)_{[3]}$

(10) $\sim^{3} \neg(B b \supset F b) \quad 1 \quad \mathrm{UI}^{3} \quad-$

(11) $B b \supset F b \quad 10 \quad \mathrm{CDN} \quad \neg(B b \supset F b)_{[3]}$

(12) $\sim^{2} \neg(P a \supset \neg F a) \quad 2 \quad \mathrm{UI}^{2} \quad-$

(13) $P a \supset \neg F a \quad 12 \quad \mathrm{CDN} \quad \neg(P a \supset \neg F a)_{[2]}$

(14) $\mathrm{Pa} \supset \mathrm{Ba} \quad 5 \quad \mathrm{UI}^{0} \quad$ -

(15) $\mathrm{Fb} \quad 6,11 \quad \mathrm{MP} \quad \neg(B b \supset F b)_{[3]}$

(16) $\mathrm{Ba} \quad 7,14 \quad \mathrm{MP} \quad-$

†(17) $\mathrm{Fa} \quad 16,9 \quad \mathrm{MP} \quad \neg(B a \supset F a)_{[3]}$

(18) $\neg F a \quad 7,13 \quad$ MP $\quad \neg(P a \supset \neg F a)_{[2]}$

$F b$ at line (15) is finally derived. Line (17) is marked in view of MR2 and line (18). $\neg F a$ at line (18) is finally derived. If we continue the proof, we can derive $\sharp^{3} \neg(B a \supset F a)$ with $\neg(P a \supset \neg F a)_{[2]}$ as fifth element; hence in view of RM line (9) has to be marked. The reader can verify that from these premises $\left(\forall^{0} x\right)\left(\sharp^{1} \neg(M x \supset F x) \vee \sharp^{2}(P x \supset \neg F x) \vee \neg M x\right)$ is derivable, and hence also $\neg M a$ and $\neg M b$, whereas $F c$ is derivable when we add the premise $M c$.

\subsection{Semantics of HL2.}

The HL2-semantics is obtained from the pHL2-semantics by defining, for each $\Gamma$, a subset of the pHL2-models of $\Gamma$. Any $\Gamma$ defines a set of semantically unreliable formulas. The HL2-models of $\Gamma$ are those pHL2-models of $\Gamma$ in which only unreliable formulas behave inconsistently. The set of unreliable formulas with respect to $\Gamma$ is a subset of the factors of the minimal DEK-consequences of $\Gamma$.

Definition: If $\left.\Gamma\right|_{\mathbf{p H L 2}} \sim^{n} B\left(\right.$ whereas $\Gamma \not \nvdash_{\mathbf{p H L 2}} \sim^{m} B(1 \leq m<n)$ ), then 
$\mathrm{CP}_{\Gamma}(B)=n$. If $\Sigma=\left\{B_{1}, \ldots, B_{n}\right\}(n \geq 1)$, then $\mathrm{CP}_{\Gamma}(\Sigma)$ is the maximum of $\mathrm{CP}_{\Gamma}\left(B_{1}\right) \ldots, \mathrm{CP}_{\Gamma}\left(B_{n}\right)$.

Definition: If $B \in \Sigma, \Sigma$ is a minimal DEK-consequence of $\Gamma$, and $\mathrm{CP}_{\Gamma}(B)=$ $\mathrm{CP}_{\Gamma}(\Sigma)$, then $B$ is $\mathbf{H L 2}$-unreliable with respect to $\Gamma . \mathcal{U}(\Gamma)$ is the set of all wffs that are HL2-unreliable with respect to $\Gamma$.

Definition: Where $\mathrm{M}$ is a pHL2-model, $\mathrm{ab}(\mathrm{M})=\left\{A \mid \mathrm{v}_{\mathrm{M}}(\sharp A)=1\right\}$.

Definition: $M$ is a HL2-model of $\Gamma$ iff it is a pHL2-model of $\Gamma$ and $\mathrm{ab}(\mathrm{M}) \subseteq \mathcal{U}(\Gamma)$.

Definition: $\Gamma=\mathbf{H L 2} A$ iff $A$ is true in all HL2-models of $\Gamma$.

\subsection{Metatheory of HL2.}

The Soundness and Strong Completeness Theorems of HL2 are analogous to those of HL1.

\subsection{Concluding Remarks.}

(i) HL2 is a logic in which most wanted consequences are finally derivable from a set of premises some of which are UQFs with (predictable or unpredictable) exceptions, whereas most unwanted are not. ${ }^{23}$ Of the two halves of an inconsistency, the one derived from the least preferred information is not finally derivable. The power of HL2 lies in its strategy. In comparison to Consistency-Based Logics, such as Default Logic, that try to resolve these kinds of inconsistencies by anticipating the exceptions (in a default), ${ }^{24}$ HL2 allows for $\sim^{n}$-inconsistencies and conditionally derived $\neg$ inconsistencies within the process (i.e. within the HL2-proofs), and resolves the $\neg$-inconsistencies when they occur. In my opnion, the most interesting property of HL2 is that exceptions need not te be known beforehand. Its dynamic proof procedure allows for the introduction of new premises at any

\footnotetext{
${ }^{23}$ From the premises $\{$ Tweety is a penguin, birds fly, penguins do not fly, and penguins are birds\}, HL2 derives that Tweety flies, and all other individuals in the domain do not fly. This is a result that is worth being compared with the results of Circumscription (Parallel Predicate Circumscription, Abnormality Theories, Prioritized Circumscription (confer the proof in Section 4.2.1); see, e.g., [5] pp. 12-21. HL2 however, is not able to conclude from "All sailors are male and have a beard" and "Sailor Popeye has no beard", that "Popeye is a man". In such cases, the easiest solution is to write two UQFs instead of one UQF containing a conjunction. (For instance: "All sailors are male" and "All sailors have a beard".)

${ }^{24}$ See, e.g., [5] pp. 39-64. Note that HL2 derives that Paul likes wine, from $\{$ Paul is Italian or French, Italians like wine, French like wine $\}$. Also HL2 does not derive that one has a usable left and a usable right arm when one has a broken left or broken right arm.
} 
stage of the proof, whereas the introduction of new premises possibily leads to a different consequence set.

(ii) It is easily seen that (a) is a pHL2-theorem:

(a) $\left(\forall^{n} \alpha\right) A \equiv(\forall \alpha)\left(\sharp^{n} \neg A \vee A\right)$

It is indeed possible to write every exceptional UQF as a classical UQF. In view of this theorem, it can easily be proven that pHL2 (and hence also HL2) has an interesting transitivity rule:

$$
\begin{aligned}
& \left(\forall^{n} \alpha\right)(A(\alpha) \supset B(\alpha)) \\
& \left(\forall^{m} \alpha\right)(B(\alpha) \supset C(\alpha)) \\
& (\forall \alpha)\left(\sharp^{n} \neg(A(\alpha) \supset B(\alpha)) \vee \sharp^{m} \neg(B(\alpha) \supset C(\alpha)) \vee(A(\alpha) \supset C(\alpha))\right)
\end{aligned}
$$

This property of pHL2 can be very useful in the reconstruction of other non-monotonic systems. ${ }^{25}$

\section{References}

[1] Batens, Diderik: "Dynamic Dialectical Logics", in G. Priest, P. Routley \& J. Norman (eds.) Paraconsistent Logic. Essays on the Inconsistent. München, pp. 187-217.

[2] Batens, Diderik: "Inconsistency-adaptive Logics and Non-monotonic Logics", Logique et Analyse 145, March 1994.

[3] Batens, Diderik: "Functioning and Teaching of Adaptive Logics." in van Benthem, Grootendorst, van Eemeren and Veltman (eds.) Logic and Argumentation. North-Holland 1996.

[4] Batens, Diderik: "Inconsistency-Adaptive Logics." in Ewa Orlowska (ed.) Essays Dedicated to the Memory of Helena Rasiowa. Heidelberg, New-York, Physica Verlag, Springer, 1998, pp. 445-472.

[5] Brewka, G., Dix, J. and Konolige, K., "Nonmonotonic Reasoning. An Overview. CSLI Publications, Stanford California, 1997.

[6] De Clercq, Kristof: "Two New Strategies for Inconsistency-adaptive logics." (to appear).

[7] Moutafakis, Nicholas, J. "The Logics of Preference", Episteme 14, D. Reidel Publishing Company, Dordrecht, Boston, Lancaster, Tokyo, 1987.

\footnotetext{
${ }^{25} \mathrm{I}$ hope to establish this in forthcoming papers.
} 